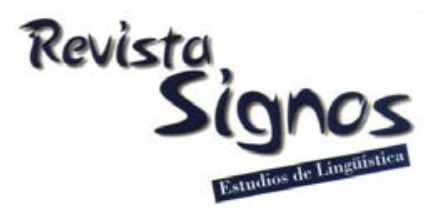

\title{
Variaciones lingüístico-discursivas en narrativas escritas por alumnos de nivel primario en Norpatagonia, Argentina ${ }^{1}$
}

\section{Linguistic-discursive variations in written narratives by elementary school children in Northern Patagonia}

\author{
María Sol \\ Iparraguirre \\ CONICET \\ UNIVERSIDAD NACIONAL DEL COMAHUE \\ ARGENTINA \\ msoliparraguirre@gmail.com
}

\author{
Nora Baccalá \\ UNIVERSIDAD NACIONAL DEL COMAHUE \\ ARGENTINA \\ norabaccala@gmail.com
}

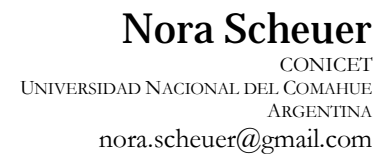

Nora Scheuer COMAHUE nora.scheuer@gmail.com

Recibido: 17/IV/2015 / Aceptado: 14/III/2016

\section{Resumen}

En este trabajo estudiamos narrativas escritas individualmente en clase y sobre papel de 54 alumnos de tercer y séptimo grado de escuelas primarias con diferencias socioeducativas en Norpatagonia, Argentina. Buscamos conocer los variados modos en que estos alumnos resuelven la producción narrativa escrita atendiendo tanto al ajuste a las prescripciones normativas de la escritura, y a las pautas que delinean el esquema narrativo, como a la elección de alternativas habilitadas por la gramática y las convenciones del género. Categorizamos los textos según tres niveles lingüísticos: textual (unidad de análisis: el texto), lexicogramatical y morfo-ortográfico (unidad de análisis: la palabra). Para estudiar las asociaciones entre las categorías definidas en cada nivel y el grado/escuela de los niños, aplicamos procedimientos de la estadística descriptiva multivariada. Los resultados mostraron una progresión evolutivo-educativa en los niveles textual y morfo-ortográfico, y patrones estilísticos en el nivel lexicogramatical, asociados a aspectos socioculturales. En conjunto, estos resultados dieron cuenta de una variedad de grados y formas de apropiación de la escritura narrativa que emergen de los modos en que los niños abordan el doble desafío de ajustarse a prescripciones y de explorar y explotar un conjunto de opciones.

Palabras Clave: Escritura, narrativas, alumnos de nivel primario, niveles lingüísticos, variación lingüística. 


\begin{abstract}
In this paper we study the written narratives used by 54 third- and seventh graders in elementary schools with different socio-educational characteristics in Northern Patagonia, Argentina, when writing individually, on paper, and during class, a text of their choice. We aim to capture the various ways in which these students solve the narrative production, considering the adjustment to specific prescriptions for written language and to conventional features of narrative structure, as of the lexicogrammatical choices according to grammar and genre. We categorized the 54 texts according to three linguistic levels: textual (unit of analysis: text), lexicogrammatical, and morpho-orthographic (unit of analysis: word). We applied diverse techniques of Multivariate Descriptive Statistics, in order to capture associations between categories in each linguistic level and children's grade/school. Results showed socio-educational trends at the textual and morpho-orthographic levels, and stilistic patterns at the lexicogrammatical level, related to socio-cultural traits. In sum, these results account for a variety of degrees and ways of appropriating narrative writing, which emerge from the double perspective of adjustment to prescriptions, as well as exploration and exploitation of a range of options.
\end{abstract}

Key Words: Writing, narratives, elementary school children, linguistic levels, literacy.

\title{
INTRODUCCIÓN
}

A través de distintas culturas, la narrativa constituye una poderosa herramienta de organización de la experiencia, el pensamiento y la memoria (Labov, 1972; Donald, 1993; Bruner, 2002; Nelson, 2014), y de socialización (Miller, Koven \& Lin, 2011). La apropiación del esquema narrativo favorece el establecimiento de conexiones consigo mismo, con otros y entre distintos tiempos, y favorece el despliegue de la singularidad del narrador en el marco de una elaboración discursiva delineada culturalmente (Bruner, 1991; Ochs \& Capps, 1996). Debido a su alta imbricación cultural y a la diversidad de funciones y situaciones en las que es utilizada, en este trabajo enfocamos sobre la narrativa como un espacio de problemas en el que operan dos orientaciones: una prescriptiva, que regula el uso de la lengua escrita en cierta situación sociodiscursiva, y otra electiva, vinculada al rango de opciones lingüístico-discursivas disponibles para el escritor en el marco de las convenciones culturales en las que se inscriba.

\section{Marco teórico}

Típicamente, una narrativa constituye el relato de acciones realizadas intencionalmente por ciertos personajes en determinados escenarios, utilizando ciertos medios de acuerdo con la finalidad perseguida. Estas vicisitudes incorporan algún tipo de desviación o elemento irruptor que aporta la intriga necesaria para que los sucesos ameriten ser narrados y se organizan según una sucesión temporal y relaciones semánticas de tipo lógico-causal (Stein \& Glenn, 1979; Bruner, 1991; Adam, 1992; Nelson, 1996; Klein, 2007). 
Las primeras narrativas de los niños (usualmente alrededor de los dos o tres años de edad) suelen relacionar dos eventos en una secuencia temporal. Progresivamente, los niños articulan más eventos con cierta organización temporo-causal explícita, conforme una jerarquía de relevancia temática y una estructura de acción en una trama explícitamente motivada (Berman \& Slobin, 1994; Nelson, 1996; Ochs \& Capps, 1996; Peterson, 2001; Miller et al., 2011). En este proceso los adultos suelen jugar un rol activo al ayudar al niño a formular verbalmente lo que intenta comunicar, en torno a la elección de los tópicos, a transmitir información con precisión, identificar los roles sociales asumidos por los participantes, organizar temporo-causalmente las acciones y dar cuenta de su posición personal respecto de lo narrado (Miller et al., 2011; Rosemberg, Silva \& Stein, 2011). Cuando los niños comienzan a producir narrativas escritas en papel, se enfrentan además a demandas vinculadas al despliegue gráfico de la lengua de forma que sea decodificable, así como al desafío de elegir qué contar, generar la trama narrativa y desplegarla de principio a fin con coherencia y cohesión.

Ya en sus tempranas producciones de textos narrativos escritos, los niños ponen en juego recursos de distintos niveles de la lengua escrita -textual, lexicogramatical, morfológico y ortográfico- (Fitzgerald \& Shanahan, 2000; Snow, 2006; Tolchinsky, 2006). La recursividad de los niveles de la lengua escrita explica la constante interacción entre elementos y estructuras de diferentes grados de complejidad (van Dijk \& Kintsch, 1983), y requiere que el escritor permanentemente conjugue el uso de convenciones socioculturales y el despliegue de elecciones personales. A nivel textual, se manifiestan aspectos composicionales de la estructura narrativa vinculados a las características de la situación comunicativa y a su interpretación por parte de quien escribe. Ello requiere elegir el tipo textual y seleccionar y organizar los componentes del esquema narrativo de acuerdo con convenciones de la estructura narrativa (Bajtín, 1985; Nelson, 1996). El nivel textual es realizado por elementos y estructuras del nivel lexicogramatical, que habilitan un ámbito de elección en el marco de las opciones disponibles para el escritor, según las reglas de combinación sintáctico-semánticas de la lengua en cuestión (Halliday, 1994; Givón, 2001). A su vez, el nivel lexicogramatical es realizado en la lengua escrita por elementos de orden morfo-ortográfico, regulado por estrictas normas de construcción de las palabras en cuanto a sus formantes morfológicos y a la representación gráfica de los sonidos de la lengua, así como por normas de diferente grado de prescripción para organizar los fragmentos textuales mediante signos de puntuación (Bosque \& Demonte, 1999). Para los aprendices, el margen de elección en este nivel solo se constata en cierta medida en la puntuación, en tanto que en los aspectos morfológicos y fonográficos impera la prescripción.

Aun con un dominio incipiente de la escritura, al escribir textos narrativos los niños se sitúan en un espacio de problemas (Simon \& Hayes, 1979) donde dos orientaciones se encuentran en tensión: una prescriptiva y una electiva (ver Figura 1). 


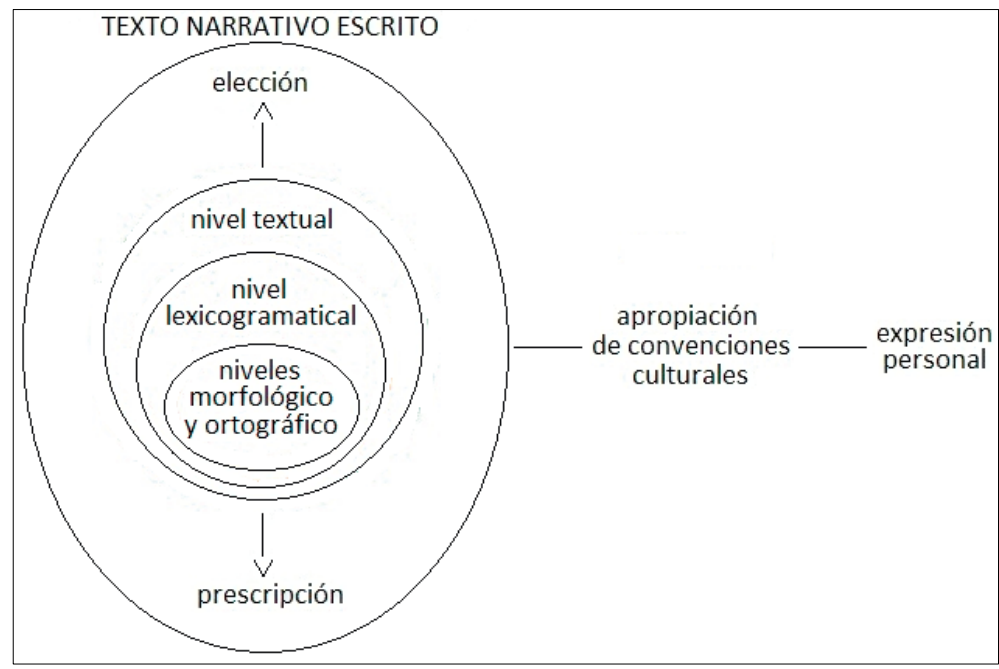

Figura 1. Niveles de la lengua escrita considerando elecciones y prescripciones en un marco narrativo.

La orientación prescriptiva atiende al ajuste a las pautas que regulan el uso de la lengua escrita y a las convenciones que caracterizan el género, habilitando que un texto narrativo escrito pueda ser decodificado y comprendido, mientras que la orientación electiva atiende a la exploración y explotación del horizonte de alternativas habilitadas por la gramática. La disponibilidad de opciones lingüístico-discursivas pertinentes y relevantes al escribir narrativas varía para cada escritor, sea experto o aprendiz, lo que habilita distintos patrones (Biber, 1988) o estilos composicionales, en los que convergen la gramática y la estilística (Bajtín, 1985; Adam, 1999; Iparraguirre, 2013).

Los estudios de narrativas escritas por niños en edad escolar han analizado principalmente la calidad textual a uno o dos niveles lingüísticos. A nivel textual, han considerado componentes de la estructura narrativa y establecimiento de coherencia y cohesión (Kamberelis, 1999; Salvador Mata, 1999; García Guzmán \& Salvador Mata, 2012) y ocasionalmente el tópico (Peterson, 2001). A nivel lexicogramatical, cantidad y diversidad léxica, uso de conjunciones, y tipo y cantidad de estructuras subordinadas (Álvarez, 1996; Junco, 1999; Beers \& Nagy, 2011). Y a nivel morfo-ortográfico, uso de puntuación, delimitación gráfica de la palabra y errores en la correspondencia grafofónica (Álvarez, 1996; Ferreiro \& Zucchermaglio, 1996; Sotomayor, Molina, Bedwell \& Hernández, 2013). La mayoría de estos estudios abordó el género narrativo como un medio para comparar niveles de logro en el aprendizaje general de la escritura en poblaciones consideradas en forma dicotómica: rendimiento escolar, presencia/ausencia de necesidades educativas especiales (Montague, Maddux \& Dereshiwsky, 1990; Lane \& Lewandowski, 1994; Montague, Salvador Mata, 1999), origen étnico o socioeconómico (Álvarez, 1996; Ferreiro \& Zucchermaglio, 1996; 
García Guzmán \& Salvador Mata, 2012; Sotomayor et al., 2013), o género (Knudson, 1995; Peterson, 2001).

En cuanto al nivel textual, se encontró que durante los primeros años de la escolarización los niños creaban narrativas escritas relativamente apropiadas al género y con cierta estabilidad en la estructura esquemática desde la infancia hasta la adolescencia (Christie \& Derewianka, 2008). A medida que los alumnos avanzan en edad y grado escolar, hay consenso en que se amplían los recursos lingüísticos utilizados. Así, en el nivel lexicogramatical, con el avance evolutivo-educativo, los niños estudiados comenzaron a producir nominalizaciones y a expandir las formulaciones por medio de la explotación de frases nominales, verbales y preposicionales, y también adverbios (Christie \& Derewianka, 2008). Mientras en los primeros años de la escolarización primaron las conexiones copulativas o temporales, niños de entre 9 y 12 años tendieron a utilizar mayor variedad de conectores y con mayor precisión (Álvarez, 1996; Christie \& Derewianka, 2008). A nivel morfoortográfico, el avance con la escolarización se registró especialmente en niños que provienen de sectores socioeconómicos medios y altos (Álvarez, 1996; Ferreiro \& Zucchermaglio, 1996; Sotomayor et al., 2013).

Conocer mejor los recursos que alumnos de primaria despliegan en diversos niveles lingüísticos al escribir narrativas en diferentes contextos socioeducativos resulta de interés por varios motivos. El más evidente radica en la importancia tanto de la narrativa como de la escritura en el desarrollo cognitivo y en la participación social de los niños. Otro es la potencialidad de un enfoque multi-nivel para captar mejor las diferentes demandas involucradas en la escritura de narrativas y los avances de los niños en la apropiación de este género de la escritura. Por último, la constatación de variaciones evolutivo-educativas y socioculturales en los modos en que los niños resuelven esta tarea puede ampliar la mirada de educadores y lingüistas acerca de los niños como aprendices y escritores.

En este trabajo nos proponemos conocer los variados modos en que alumnos de nivel primario con diferentes características socioeducativas en un mismo distrito escolar resuelven la tensión entre las orientaciones prescriptiva y electiva al escribir un texto narrativo. En particular, buscamos conocer cómo los niños ponen en juego ambas orientaciones (i) a nivel textual y (ii) lexicogramatical; así como (iii) su apropiación de las prescripciones normativas a nivel morfo-ortográfico. Además, nos interesa registrar (iv) la variación en dichos aspectos según dos momentos claves en la consecución del nivel primario y según diferentes características socioculturales.

En la zona en la que se llevó a cabo el estudio, el nivel primario comprende siete años y se organiza en dos ciclos. El primer ciclo abarca desde primero hasta tercer grado ( 6 a 8 años de edad) y el segundo ciclo abarca desde cuarto hasta séptimo grado (9 a 12 años). Debido a ello, decidimos centrarnos en los años de culminación de los 
mismos: tercer y séptimo grado. En cuanto a las características socioculturales de los alumnos, a fin de considerar un espectro sociocultural y educativo amplio tuvimos en cuenta una conjunción de criterios para la selección de cuatro escuelas: ubicación de la escuela en la geografía urbana, gestión y organización escolar, nivel educativo de los padres y nivel socioeconómico.

\section{Metodología}

\subsection{Participantes}

Participaron del estudio alumnos de tercer y séptimo grado de cuatro escuelas primarias del distrito escolar de Bariloche (115.000 habitantes), Norpatagonia, Argentina. Las escuelas fueron seleccionadas atendiendo a criterios de diversidad sociocultural y educativa, en particular en relación a tres parámetros de impacto sobre la alfabetización de los niños (Ferreiro \& Teberosky, 1979; Maimone \& Edelstein, 2004; Ministerio de Cultura y Educación de la Nación, 2010): distancia e integración geográfico-social respecto del centro urbano; régimen educativo; y características socioeconómicas y culturales del alumnado. Esta información se obtuvo en reuniones con informantes claves de la comunidad educativa local y con supervisores y equipos directivos de las escuelas, durante las cuales presentamos además el estudio, a fin contar con el consentimiento informado por parte de supervisores y autoridades. Dichos establecimientos fueron:

1. Escuela Céntrica (EC). Privada y de doble jornada. Situada en un barrio céntrico de la ciudad de Bariloche. Alumnos de estratos socioeconómicos medios y altos.

Los siguientes establecimientos eran de gestión estatal. Los padres contaban generalmente con el nivel primario completo.

2. Escuela Periférica (EP). Jornada simple. Situada en un barrio periféricomarginado de la ciudad con altos índices de pobreza y desocupación. Grados con alumnos con uno a tres años de diferencia de edad entre ellos (debido a repitencia).

3. Escuela Rural (ER). Jornada simple. Situada en un paraje alejado de la ciudad, con grupos escolares monogrados o que reúnen dos grados sucesivos.

4. Escuela-Hogar (EH). Jornada simple con apoyo escolar y pernocte de lunes a viernes. Ubicada en el campo. Grupos escolares de dos o tres grados sucesivos.

Dada la variabilidad en el número de alumnos en cada grado (entre 4 y 22 alumnos) se realizó una selección buscando que ningún grado superara en más del doble la cantidad de alumnos de los grados más pequeños, de modo de equilibrar el peso relativo de cada alumno sin reducir excesivamente la cantidad de participantes (ver Tabla 1). 
Tabla 1. Descripción de los grados escolares y de los alumnos seleccionados.

\begin{tabular}{|c|c|c|c|c|c|c|}
\hline & \multirow{3}{*}{$\begin{array}{l}\text { Total } \\
\text { grado }\end{array}$} & \multicolumn{5}{|c|}{ Alumnos seleccionados } \\
\hline & & \multicolumn{3}{|c|}{ Cantidad } & \multicolumn{2}{|c|}{ Edad } \\
\hline & & Niñas & Niños & Total & Promedio & $\begin{array}{c}\text { Desviación } \\
\text { estándar }\end{array}$ \\
\hline \multicolumn{7}{|l|}{$3^{\circ} \mathrm{gr}}$. \\
\hline Escuela Céntrica (EC3) & 17 & 3 & 5 & 8 & 8 años 7 meses & 3 meses \\
\hline Escuela Periférica (EP3) & 6 & 1 & 5 & 6 & 9 años 2 meses & 1 año 5 meses \\
\hline Escuela Rural (ER3) & 5 & 1 & 4 & 5 & 9 años & 8 meses \\
\hline Escuela-Hogar (EH3) & 9 & 6 & 2 & 8 & 8 años 9 meses & 1 año 1 mes \\
\hline \multicolumn{7}{|l|}{$7^{\circ}$ gr. } \\
\hline Escuela Céntrica (EC7) & 22 & 5 & 3 & 8 & 12 años 7 meses & 2 meses \\
\hline Escuela Periférica (EP7) & 16 & 6 & 2 & 8 & 13 años 6 meses & 9 meses \\
\hline Escuela Rural (ER7) & 7 & 3 & 4 & 7 & 13 años 4 meses & 10 meses \\
\hline Escuela-Hogar (EH7) & 4 & 0 & 4 & 4 & 13 años 6 meses & 10 meses \\
\hline
\end{tabular}

Nota: Entre paréntesis se informan los códigos con los que se identifican los grados en los

Resultados.

Así, una vez retiradas las producciones de alumnos con necesidades educativas especiales (que no fueron objeto de estudio), en los grados con menos de ocho alumnos se retuvo el grado completo, mientras que en los grados más numerosos se realizó una selección al azar de ocho alumnos, conservando la proporción original entre mujeres y varones.

\subsection{Procedimiento y materiales}

Tras dos visitas de familiarización a cada grado, una de las autoras propuso oralmente a todos los alumnos que escribieran en forma individual una historia de su elección:

"Quiero que me cuenten una historia. [Para promover la producción textual y el posicionamiento de los alumnos como autores, agregó:] Puede ser una historia que les hayan contado, en sus casas, por ejemplo, que puede ser real o no. Alguna historia que a ustedes les guste, o los asuste un poco, o que les hayan contado muchas veces... Lo que ustedes quieran."

Luego entregó a los alumnos hojas rayadas y biromes (lápices pasta/bolígrafos). La actividad ocupó una hora de la asignatura Lengua y se realizó durante el segundo trimestre del año. Los textos fueron transcritos digitalmente respetando la formulación y ortografía originales.

\subsection{Niveles, dimensiones y categorías de análisis}

Para conocer las elecciones realizadas por los alumnos en este espacio textual y su ajuste a las convenciones de la lengua escrita y del género narrativo, consideramos los niveles presentados en la Introducción: textual, lexicogramatical y morfo-ortográfico (ver Tabla 2). 
Tabla 2. Dimensiones, alcance y unidad de análisis para los tres niveles.

\begin{tabular}{|c|c|c|c|}
\hline Niveles & Dimensiones & Alcance & Unidad \\
\hline \multirow[t]{5}{*}{ Textual } & Tópico & Clasificación temática global. & El texto completo. \\
\hline & \multirow[t]{3}{*}{$\begin{array}{l}\text { Estructuración } \\
\text { narrativa }\end{array}$} & $\begin{array}{l}\text { Explicitación de los componentes } \\
\text { de la unidad temática. }\end{array}$ & \multirow[t]{3}{*}{ Secuencias textuales. } \\
\hline & & $\begin{array}{l}\text { Inclusión de componentes de la } \\
\text { unidad de acción. }\end{array}$ & \\
\hline & & Secuencialidad temporo-causal. & \\
\hline & $\begin{array}{l}\text { Expresiones } \\
\text { narrativas }\end{array}$ & $\begin{array}{l}\text { Explicitación del comienzo o el fin } \\
\text { de la historia o inclusión de } \\
\text { fórmulas características de la } \\
\text { narrativa. }\end{array}$ & Secuencias textuales. \\
\hline \multirow[t]{4}{*}{$\begin{array}{l}\text { Lexico- } \\
\text { gramatical }\end{array}$} & Categorial & $\begin{array}{l}\text { Clasificación de las palabras según } \\
\text { su clase léxica. }\end{array}$ & Palabras ortográficas. \\
\hline & \multirow[t]{2}{*}{ Sintáctica } & $\begin{array}{l}\text { Función sintáctica a la que } \\
\text { contribuyen las palabras. }\end{array}$ & Mayoría de las palabras. \\
\hline & & $\begin{array}{l}\text { Funcionamiento sintáctico de los } \\
\text { nexos. }\end{array}$ & $\begin{array}{l}\text { Todas las palabras que } \\
\text { articulan partes del texto. }\end{array}$ \\
\hline & Semántica & $\begin{array}{l}\text { Rol semántico al que contribuyen } \\
\text { las palabras. }\end{array}$ & Mayoría de las palabras. \\
\hline \multirow[t]{3}{*}{$\begin{array}{l}\text { Morfo- } \\
\text { ortográfico }\end{array}$} & de Puntuación & $\begin{array}{l}\text { Uso de distintos signos de } \\
\text { puntuación. }\end{array}$ & Signos de puntuación. \\
\hline & Morfológica & $\begin{array}{l}\text { Delimitación de los límites } \\
\text { formales de la palabra, errores de } \\
\text { concordancia en género y número, } \\
\text { y total de palabras producidas. }\end{array}$ & Palabras ortográficas. \\
\hline & Fonografémica & $\begin{array}{l}\text { Errores en el ajuste ortográfico de } \\
\text { la palabra y en la correspondencia } \\
\text { fononema-grafema. }\end{array}$ & Grafemas. \\
\hline
\end{tabular}

\subsubsection{Nivel textual}

Analizamos para cada texto tópico de la narración, estructuración narrativa (Labov, 1972; Adam, 1992; Klein, 2007) y expresiones formularias narrativas (Klein, 2007), de acuerdo con las categorías presentadas en la Tabla 3. En el nivel textual se realizó un control interjuez con un segundo investigador para el 30\% de los textos. Todos los casos de desacuerdo fueron discutidos hasta arribar al consenso. 
Tabla 3. Nivel textual: Dimensiones y categorías de análisis.

\begin{tabular}{|c|c|c|}
\hline & mensiones & Categorías \\
\hline Tópico de & narración & Relatos mágicos, paranormales o de ciencia ficción (T-MPCf). \\
\hline & & Relatos románticos, heroicos o de una picardía (T-RHP). \\
\hline & & $\begin{array}{l}\text { Relatos testimoniales referidos al lugar en el que se desarrollan los } \\
\text { alumnos (T-TLug). }\end{array}$ \\
\hline & & $\begin{array}{l}\text { Relatos testimoniales referidos a aspectos personales, familiares o } \\
\text { comunitarios (T-TPFC). }\end{array}$ \\
\hline & & Relatos aleccionadores (T-Alec). \\
\hline & & $\begin{array}{l}\text { Otro tipo de relatos: tópicos mixtos, canciones y textos inconclusos } \\
\text { (T-Otros). }\end{array}$ \\
\hline $\begin{array}{l}\text { Estructu- } \\
\text { ración }\end{array}$ & $\begin{array}{l}\text { Estrategias de } \\
\text { introducción y }\end{array}$ & $\begin{array}{l}\text { Textos que anticipan el relato y/o presentan una situación generadora } \\
\text { del mismo (EstIC). }\end{array}$ \\
\hline narrativa & $\begin{array}{l}\text { cierre del relato y } \\
\text { elementos de la }\end{array}$ & $\begin{array}{l}\text { Textos que incluyen una situación inicial, un conflicto y una situación } \\
\text { final (SI-C-SF). }\end{array}$ \\
\hline & unidad de acción & $\begin{array}{l}\text { Textos que anticipan el relato y/o presentan una situación generadora } \\
\text { del mismo y que incluyen una situación inicial, un conflicto y una } \\
\text { situación final (EstIC/SI-C-SF). }\end{array}$ \\
\hline & & $\begin{array}{l}\text { Textos que anticipan el relato y/o presentan una situación generadora } \\
\text { del mismo y que incluyen una situación inicial y una situación final } \\
\text { (EstIC/SI-SF). }\end{array}$ \\
\hline & & $\begin{array}{l}\text { Textos que no incluyen ninguno de los elementos previos (Est/UA- } \\
\mathrm{N}) \text {. }\end{array}$ \\
\hline & $\begin{array}{l}\text { Explicitación de la } \\
\text { unidad temática }\end{array}$ & $\begin{array}{l}\text { Relatos en los que entidades animadas realizan acciones en un tiempo } \\
\text { pasado y en los que el autor introduce a los personajes y sitúa el } \\
\text { tiempo y/o el espacio de las acciones (UT1). }\end{array}$ \\
\hline & & $\begin{array}{l}\text { Relatos en los que entidades animadas realizan acciones en un tiempo } \\
\text { pasado y en los que el autor o bien introduce a los personajes, o bien } \\
\text { sitúa el tiempo y/o el espacio de las acciones (UT2). }\end{array}$ \\
\hline & & $\begin{array}{l}\text { Relatos en los que entidades animadas realizan acciones en tiempo } \\
\text { presente, y en los que los autores alternativamente incluyen acciones } \\
\text { en pasado, introducen a los personajes o sitúan el tiempo y/o el } \\
\text { espacio de las acciones (UT3). }\end{array}$ \\
\hline & & $\begin{array}{l}\text { Relatos en los que se encuentra uno de los elementos previamente } \\
\text { mencionados o ninguno (UT4). }\end{array}$ \\
\hline & Secuenciación & En el texto predomina la cópula de secuencias (STC-Cop). \\
\hline & $\begin{array}{l}\text { temporo-causal de } \\
\text { las acciones }\end{array}$ & $\begin{array}{l}\text { El texto presenta orden temporal cronológico y secuenciación causal } \\
\text { de las acciones (STC-TC). }\end{array}$ \\
\hline & & $\begin{array}{l}\text { El texto presenta orden temporal cronológico y secuenciación causal } \\
\text { de las acciones con algunos quiebres u omisiones (STC-TCQ). }\end{array}$ \\
\hline & & $\begin{array}{l}\text { En el texto predomina la cópula de secuencias con algunos quiebres u } \\
\text { omisiones (STC-CopQ). }\end{array}$ \\
\hline & & El texto no presenta ninguno de los elementos previos (STC-N). \\
\hline Expresion & formularias & El texto incluye fórmulas de inicio y de cierre del relato (F-IC). \\
\hline narrativas & & El texto incluye sólo fórmulas de inicio (F-I). \\
\hline & & El texto incluye sólo fórmulas de cierre (F-C). \\
\hline & & El texto no incluye fórmulas ni de inicio ni de cierre (F-N). \\
\hline
\end{tabular}

Nota: Entre paréntesis se incluyen los códigos que identifican cada categoría en Resultados.

\subsubsection{Nivel lexicogramatical}

Las categorías de este nivel integran tres dimensiones: clase léxica (Bosque \& Demonte, 1999; González Calvo, 1999), sintáctica (Bosque \& Demonte, 1999; Givón, 2001) y semántica (Dowty, 1991; Givón, 2001; Halliday, 1994) (ver Tabla 4). En las 
tres dimensiones fueron registradas para cada texto las frecuencias absolutas de palabras en cada categoría. En la dimensión clase léxica se consideraron conjuntamente adjetivos y determinativos por su baja frecuencia.

Tabla 4. Nivel lexicogramatical.

\begin{tabular}{|c|c|c|}
\hline \multicolumn{2}{|c|}{ Dimensiones } & Categorías \\
\hline \multirow{7}{*}{\multicolumn{2}{|c|}{ Clase léxica }} & Sustantivo (SUST) \\
\hline & & Pronombre (PRO) \\
\hline & & Adjetivo/determinativo (ADJ/DET) \\
\hline & & Verbo (VERBO) \\
\hline & & Adverbio (ADV) \\
\hline & & Conjunción (CONJ) \\
\hline & & Preposición (PRE) \\
\hline \multirow[t]{6}{*}{ Sintáctica } & \multirow{4}{*}{$\begin{array}{l}\text { Función } \\
\text { sintáctica }\end{array}$} & Sujeto (FX-Suj) \\
\hline & & Complemento directo (FX-CD) \\
\hline & & Complemento indirecto (FX-CI) \\
\hline & & $\begin{array}{l}\text { Complemento circunstancial/de régimen preposicional (FX- } \\
\text { CC/RP) }\end{array}$ \\
\hline & \multirow[t]{2}{*}{ Nexo } & Coordinante (FX-NCoord) \\
\hline & & Subordinante (FX-NSub) \\
\hline \multirow{7}{*}{\multicolumn{2}{|c|}{ Semántica }} & Impulsor (RS-Imp) \\
\hline & & Paciente (RS-Pac) \\
\hline & & Experimentante/diciente (RS-Exp/Dic) \\
\hline & & Benefactivo (RS-Ben) \\
\hline & & Fenómeno/verbalización (RS-Fen/Verbaliz) \\
\hline & & Locativo espacial/temporal (RS-Loc esp/temp) \\
\hline & & Manera/instrumental/comitativo (RS-Man/Inst/Comit) \\
\hline
\end{tabular}

Nota: Entre paréntesis se incluyen los códigos con los que identificamos cada categoría en Resultados.

\subsubsection{Nivel morfo-ortográfico}

Integra tres dimensiones: de puntuación (García Negroni, 2010; R.A.E., 2011), morfológica (Bosque \& Demonte, 1999) y fonografémica (García Negroni, 2010; R.A.E., 2011) (Tabla 5). Las concordancias fueron registradas como categorías nominales. En las otras dimensiones fueron registradas para cada texto las frecuencias relativas respecto del total de palabras en el texto: exactas para la puntuación y la dimensión fonografémica y con rangos para las palabras encadenadas (por ejemplo, “muchascosas") e hipersegmentadas (por ejemplo, "es pecial”). 
Tabla 5. Nivel morfo-ortográfico.

\begin{tabular}{|c|c|c|}
\hline \multicolumn{2}{|c|}{ Dimensiones } & Categorías \\
\hline \multirow{4}{*}{\multicolumn{2}{|c|}{ de Puntuación }} & Puntos (P-punto) \\
\hline & & Mayúscula (P-may) \\
\hline & & Comas (P-coma) \\
\hline & & Signos complejos (P-signos compl) \\
\hline \multirow{11}{*}{ Morfológica } & \multirow[t]{4}{*}{ Encadenamientos } & $0 \%(\mathrm{ME} 0)$ \\
\hline & & $1-2 \%($ ME1-2) \\
\hline & & 5-9\% (ME5-9) \\
\hline & & $15-74 \%$ (ME15-74) \\
\hline & \multirow[t]{3}{*}{ Hipersegmentaciones } & $0 \%(\mathrm{MH} 0)$ \\
\hline & & $1-2 \%(\mathrm{MH} 1-2)$ \\
\hline & & $3-5 \%(\mathrm{MH} 3-5)$ \\
\hline & \multirow{4}{*}{$\begin{array}{l}\text { Concordancias en } \\
\text { género y número }\end{array}$} & Ningún error de concordancia (MCn) \\
\hline & & Omisión de marcas de plural (MCo) \\
\hline & & Agregado de marcas de plural (MCa) \\
\hline & & $\begin{array}{l}\text { Omisiones o agregados de marcas de plural y errores } \\
\text { de concordancia en relaciones anafóricas o sujeto- } \\
\text { verbo }(\mathrm{MC}+)\end{array}$ \\
\hline \multirow{4}{*}{\multicolumn{2}{|c|}{ Fonografémica }} & Uso inadecuado de mayúsculas (FG-may) \\
\hline & & Uso inadecuado de diacríticos (FG-diac) \\
\hline & & Omisión de letras (FG-omis) \\
\hline & & Sustitución de letras (FG-sust) \\
\hline
\end{tabular}

Notas: Entre paréntesis se incluyen los códigos utilizados en Resultados. La categoría referida al uso de mayúsculas en la dimensión de puntuación se aplica a la marcación del inicio de la oración, en tanto que en la dimensión fonografémica se aplica exclusivamente a su uso inadecuado en nombres comunes y propios.

\subsection{Análisis estadísticos}

Para el análisis estadístico de la información se conformaron tres tablas de datos, una para cada nivel de análisis. Dichas tablas presentaron 54 filas (una por cada texto), mientras que las columnas recogieron las distintas categorías de cada dimensión (ver Tablas 3, 4 y 5).

Para analizar las asociaciones entre los 54 textos y las categorías al interior de cada dimensión, se utilizaron técnicas de la estadística descriptiva multivariada, a saber: para el nivel textual, se aplicó Análisis de Correspondencias Múltiples (Benzécri, 1976; Lebart, Morineau \& Piron, 1995), y para los niveles lexicogramatical y morfoortográfico, Análisis Factorial Múltiple Intratabla (Bécue-Bertaut \& Pagès, 2000).

En estos análisis las variables pueden ser activas o ilustrativas. Las variables activas definen los ejes factoriales, mientras que las variables ilustrativas son proyectadas $a$ posteriori sobre los mismos, y se utilizan para complementar la estructura de la información brindada por las variables activas (Crivisqui, 1993). El grado escolar fue una variable ilustrativa, con ocho categorías que combinan grado y escuela (ver Tabla 1). La variable sexo no fue considerada, por no contar en todos los grados con una población mixta (por ejemplo, EH7 se encuentra conformado únicamente por niños). 
Los resultados del Análisis Factorial Múltiple Intratabla y del Análisis de Correspondencias Múltiples se visualizan en planos factoriales, permitiendo formar grupos de asociaciones de categorías y textos. Para corroborar tales asociaciones se aplicó un Análisis de Clasificación (Lebart et al., 1995), cuyas variables son las coordenadas de los 54 textos en los principales ejes factoriales, que explican $80 \%$ o más de la variabilidad total de los datos.

Para cada grupo de asociaciones (en adelante: grupo) se realizó una descripción cualitativa en base a los textos originales completos de los alumnos más contributivos a la inercia de los primeros tres ejes. En la descripción de los grupos de los análisis textual y lexicogramatical se integraron pasajes literales de los escritos de estos alumnos a título ilustrativo. Para todos los análisis estadísticos se utilizó el programa SPAD Recherche 5.5.

\section{Resultados}

En los tres análisis realizados (uno por cada nivel) se seleccionaron los primeros tres ejes factoriales. Por razones de espacio, se presenta sólo el primer plano, proyectando en el mismo únicamente los alumnos/textos y las categorías con buena calidad de representación en tal plano. Para facilitar la lectura, los resultados de los niveles lexicogramatical y morfo-ortográfico se muestran las categorías (variables) y los textos (individuos) por separado.

\subsection{Nivel textual: $\mathbf{3}$ grupos (Figura 2)}

Grupo 1. Estos textos tienden a incorporar tópicos testimoniales referidos al lugar en el que se desarrollan los alumnos (P36: bedenelados venden alimales venden autos venden cabayos y guelles...) (T-TLug), a presentar otros tipos de textos (T-Otros), como canciones (R33: arros con lech mequiero caasar con unaseñorit de sanico la que se pategr quepaordar que ari lapuer para paiajuar), y también textos inconclusos (R34: Habia una ves una niñita que). En estos textos no suelen encontrarse los componentes específicos del esquema narrativo, tales como: secuencias que delinean la unidad temática (UT4), estrategias de comienzo y finalización de la narrativa y secuencias que explicitan la unidad de acción (Est/UA-N); o los elementos que explicitan secuencialidad temporo-causal (STC-N), tampoco incorporan por lo general expresiones específicamente narrativas para indicar el inicio o el cierre del relato (FN). Este grupo está formado principalmente (80\%) por textos de alumnos de tercer grado de las escuelas Rural y Periférica (ER3 y EP3).

Grupo 2. Reúne textos que tratan tópicos testimoniales referidos a aspectos personales, familiares o comunitarios, construyendo relatos en base a experiencias personales propias (H35: fui aun sirco mediberti mucho) (T-TPFC), mayormente en tiempo pasado, con secuencias en tiempo presente vinculadas a los propios gustos y 
percepciones (H35: me gusta ber peliculas). Involucran a familiares o conocidos de la zona (H38: mi papa hace bancos se los da ha mi mama ella los yeva ala estepa) y ocasionalmente presentan a los personajes y el tiempo/lugar de las acciones (H35: fui a zarmantin a bistar a mi primo tiene 25 anis es adulto que seanota al ejersito sellma sebastian) (UT3). Estos relatos se caracterizan además por explicitar el motivo o la situación generadora de la historia, por anticipar lo que se relatará (H38: mi hermano meconto sobre el jiete sin cabesa yo me asusto), o bien por explicitar su finalización (H38: mi historia fin) (EstIC). La construcción textual se articula principalmente a partir de conexiones copulativas (STC-Cop) que mantienen la coherencia temática pese a presentar algunos quiebres y omisiones (STC-CopQ) (H35: andu be una solabez en auto ... fui adormi en una osteria $y$ después me fui aun zologico de bolzon en bolzon vi muchascosas $\underline{y}$ opgetos $\underline{y}$ fui aun sirco ... muchas beses fui a bariloche). Integran este grupo mayoritariamente $(60 \%)$ textos de alumnos de tercer grado de la Escuela-Hogar (EH3).

Grupo 3. Este grupo reúne una variedad de relatos: mágicos, paranormales o de ciencia ficción (T-MPCf), románticos, heroicos o de picardía (T-RHP), aleccionadores (T-Alec). Se aprecia la explicitación de los personajes, especificidades de tiempo y/o lugar (UT1 y UT2) de las acciones (C76: con Ailén (una amiga) // C77: en un estudio de canal seis) y de distintos componentes de la unidad de acción según un orden prototípico (SI-C-SF). Así, estos alumnos suelen presentar la situación inicial (C79: Había una vez un príncipe que había hecho una fiesta de disfraces para encontrar una chica para casarce con ella), seguida por el conflicto que da origen a la narrativa (C79: Hasta que se encontró con una que estaba enmascarada. Bailo y bailo. Hasta que se fue y no la encontró más) y la situación final que deriva de la resolución del conflicto (C79: Hasta que la encontró. Se casaron y vivieron felices por siempre). Eventualmente incluyen el motivo, la situación generadora o la anticipación de lo que relatarán (C77: Yo voy a contar una historia que me contaron) (EstIC/SI-C-SF). La explicitación de la secuencialidad temporo-causal de las acciones (STC-TC) delinea la progresión del relato (C76: Antes de empezar... y cuando ella lo señala... empezó a gritar... terminamos empapadas). Aparecen ocasionalmente quiebres u omisiones temporo-causales (STC-TCQ), vinculados fundamentalmente a la inversión del orden de la información y al sobreuso de ciertos adverbios y construcciones adverbiales, dificultando la jerarquización de la información (por ejemplo, "ahora" o "hasta que" en oraciones consecutivas, quebrando la relación causal). Estos textos integran fórmulas que enmarcan en el género narrativo (C79: Había una vez ... y vivieron felices por siempre) (F-I y F-C). Este grupo está integrado por textos de alumnos principalmente $(75 \%)$ de la Escuela Céntrica y de séptimo grado. 


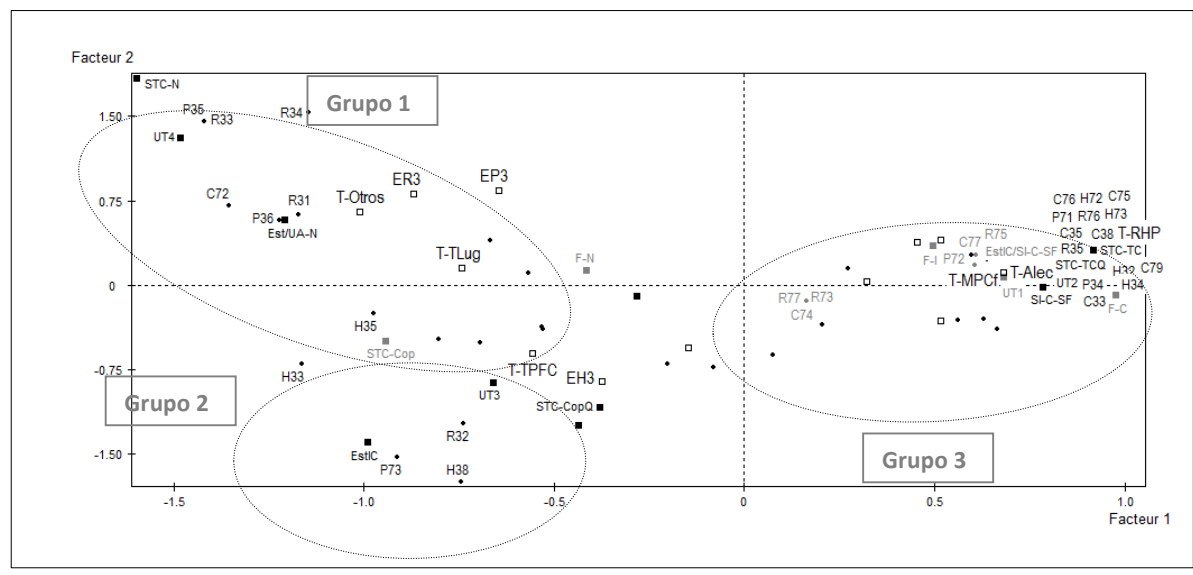

Figura 2. Nivel textual. Primer plano factorial del Análisis Factorial Múltiple Intratabla: Proyección de las categorías, los textos y los grados escolares bien representados.

Nota: Los códigos utilizados para identificar las categorías fueron presentados en la Tabla 3 y los utilizados para identificar los grados escolares fueron presentados en la Tabla 1.

\subsection{Nivel lexicogramatical: 5 grupos (Figura 3: categorías; Figura 4: textos)}

Grupo 1. Estos textos se caracterizaron por la clase léxica preposición (PRE), la función sintáctica complemento circunstancial/de régimen preposicional (FX-CC/RP) y los roles semánticos locativo espacial/temporal (RS-Loc esp/temp) y manera/instrumental/ comitativo (RS-Man/Inst/Comit). Estas diversas categorías operaron en la construcción de circunstantes de espacio y tiempo (P35: un dia // P79: en el barrio union) o manera (P35: cantaba muy bien... lo echaron a patadas... // P79: con sus hijos), y fueron realizados por frases preposicionales. Este grupo se formó mayoritariamente (55\%) por los textos de alumnos de ambos grados de la Escuela Periférica (EP3 y EP7).

Grupo 2. Estos textos se caracterizaron por las clases léxicas sustantivo (SUST) y adjetivo/determinativo (ADJ/DET), y por los roles semánticos paciente (RS-Pac) e impulsor (RS-Imp). Los grupos nominales constituyeron elementos de base para hilar el devenir de los acontecimientos (P74: la istoria: hace un par de meses en mi bario... un tiempo despues le robaron dos beses a la escuela. La primera vez le robaron la canasta de rifa... la segunda ves le robaron las computadoras y la heladera). Estos grupos nominales tienden a estar formados por un determinativo y un sustantivo, incorporando ocasionalmente un adjetivo calificativo o cuantificador. Entre los roles semánticos de estos grupos nominales, se destacan los de impulsor y paciente de las acciones y sucesos, formando cláusulas transitivas (R31: la blanquita tocó soñadores... le entregaria mi corason). Este grupo se formó principalmente $(60 \%)$ por textos de 
alumnos de tercer grado de la Escuela Rural (ER3) y de séptimo grado de la Escuela Periférica (EP7).

Grupo 3. Estos textos se caracterizaron por categorías de las tres dimensiones consideradas: la clase léxica pronombres (PRO), las funciones sintácticas complemento directo (FX-CD) y complemento indirecto (FX-CI) y los roles semánticos experimentante/diciente (RS-Exp/Dic), fenómeno/verbalización (RSFen/Verbaliz) y benefactivo (RS-Ben). En estas tramas narrativas se destacan las acciones que denotan procesos mentales o verbales. Estos alumnos codificaron las percepciones, procesos y estados mentales de los protagonistas -frecuentemente, ellos mismos-, así como el modo de acceso a estos relatos, por medio de complementos directos e indirectos (H39: ami me gusta andar a caballo / / H39: Me conto mi mama un historia). Los complementos directos de este tipo de verbos introducen verbalizaciones (R75: la chica le dijo que vaya a buscarla a su casa... la madre de la chica le dijo al hombre que su hija habia muerto) e incorporan los fenómenos experimentados (H39: conozco muchos animales), mientras que los complementos indirectos suelen codificar al destinatario de dicha verbalización (R75: la chica le dijo... la madre de la chica le dijo al hombre), con el rol de benefactivo, y fueron realizados frecuentemente por medio de pronombres. En este grupo se asocian principalmente (70\%) textos de alumnos de tercer grado de la Escuela-Hogar (EH3) y de séptimo grado de la Escuela Rural (ER7).

Grupo 4. Estos textos se caracterizaron por el uso de dos categorías: la clase léxica conjunción (CONJ) y la función sintáctica nexo coordinante (FX-NCoord). Los alumnos de este grupo avanzaron en el relato articulando las acciones por medio de la cópula de oraciones simples (C31: avia una vez... $\$ una bes vino una nave... $\underline{y}$ se llebo el cristal... y empesaron a haber emfermedades / / C38: una noche habia dos chicos... $y$ habia un perro... $\$ el perro rabioso... agarraron piedras $y$ se las tiraron, le dieron $y$ se escaparon). Este grupo se formó exclusivamente (100\%) con textos de alumnos de tercer grado de la Escuela Céntrica (EC3).

Grupo 5. Estos textos se caracterizaron por las clases léxicas adverbio (ADV) y verbo (VERBO), y la función sintáctica nexo subordinante (FX-Nsub). La construcción textual se apoyó principalmente en verbos conjugados, verboides y perífrasis, aportando distintos matices temporales y aspectuales. Las perífrasis aportan matices de significado vinculados al deber, a la intención o esfuerzo por realizar la acción, a su transcurso y a su comienzo o término (C76: Ailén me quería mostrar... estábamos jugando... empezó a gritar // C79: intentando encontrar a su chica). Los procesos verbales fueron expandidos con elementos y estructuras adverbiales de manera e instrumentales, así como por proposiciones de gerundio. La articulación textual presentó un alto grado de cohesión, con conexión de fragmentos textuales a través de adverbios (entonces) y de encabezadores de subordinación adverbial (hasta que), marcando la secuenciación temporo-causal de las acciones. Otra estrategia 
frecuente de conexión textual y expansión de la información fue la integración de cláusulas subordinadas (C76: lo que Ailén no sabía... cuando ella lo señala // C79: un príncipe que había hecho una fiesta de disfraces). Este grupo se formó exclusivamente $(100 \%)$ con textos de alumnos de séptimo grado de la Escuela Céntrica (EC7).

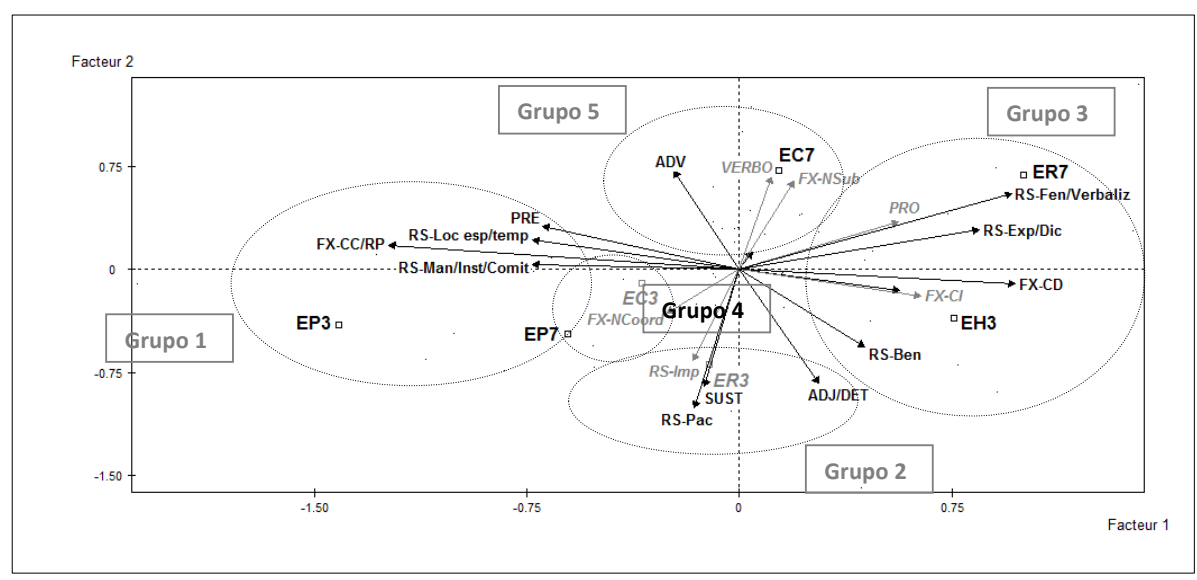

Figura 3. Nivel lexicogramatical. Primer plano factorial del Análisis de Correspondencias Múltiples: Proyección de las categorías y los grados escolares bien representados.

Nota: Los códigos utilizados para identificar las categorías fueron presentados en la Tabla 4 y los respectivos para los grados escolares en la Tabla 1.

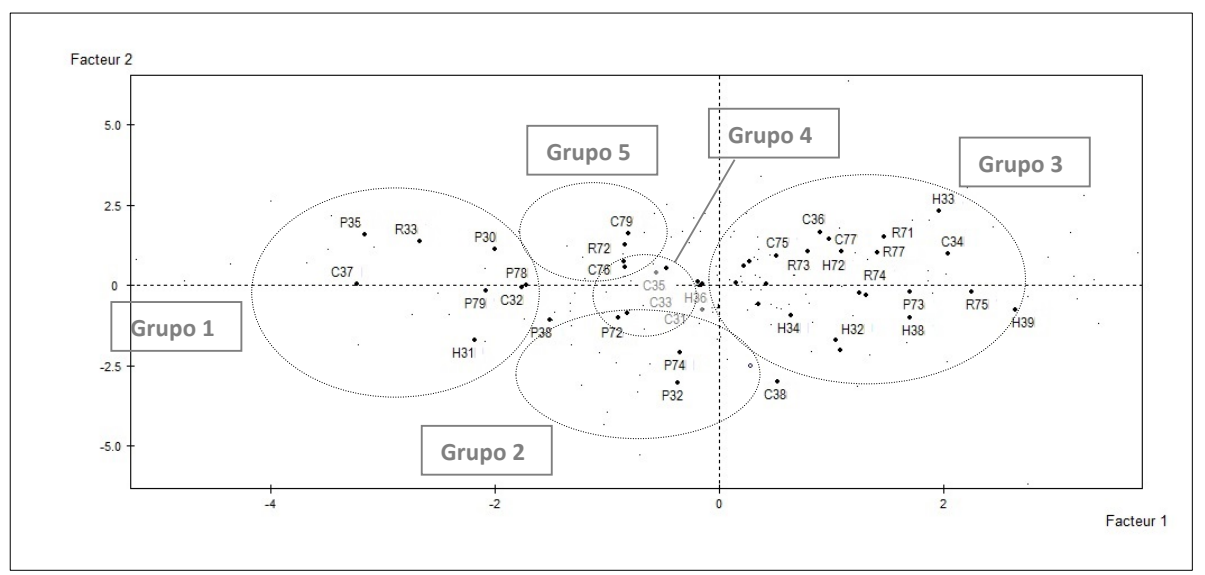

Figura 4. Nivel lexicogramatical. Primer plano factorial del Análisis de Correspondencias Múltiples: Proyección de los textos de los alumnos bien representados.

\subsection{Nivel morfo-ortográfico: $\mathbf{3}$ grupos (Figura 5: categorías; Figura 6: textos)}

Grupo 1. Estos textos se caracterizaron por categorías de la dimensión morfológica y fonografémica. En cuanto a las categorías morfológicas, son las que 
denotan las mayores dificultades para ajustar la producción de palabras a su delimitación formal, establecidas en base a los porcentajes más altos de palabras escritas con hipersegmentaciones internas (MH1-2 y MH3-5) y de palabras formando cadenas (ME15-74) respecto de las palabras totales del texto. En cuanto a la dimensión fonografémica, el grupo se caracteriza por omisiones (FG-omis) y sustituciones (FG-sust) de letras en las palabras, errores en el uso de diacríticos (FGdiac) y de la mayúscula inicial para diferenciar nombres propios de comunes (FGmay). En este grupo se asocian fundamentalmente (80\%) textos de alumnos del tercer grado de la Escuela Periférica (EP3) y del tercer grado de la Escuela Rural (ER3).

Grupo 2. Estos textos se caracterizaron exclusivamente por categorías morfológicas que muestran un mayor ajuste a la delimitación formal de la palabra, con la presencia eventual de encadenamientos respecto de las palabras totales de los textos (ME1-2) y la ausencia de hipersegmentaciones (MH0). Además caracterizan este grupo la omisión (MCo) y el agregado ( $\mathrm{MCa}$ ) de marcas para el establecimiento de concordancias de plural. Este grupo se formó con exclusivamente (100\%) con textos de alumnos de los terceros grados de la Escuela Céntrica (EC3) y Hogar (EH3), y del séptimo grado de la Escuela Rural (ER7).

Grupo 3. Estos textos se caracterizaron por el uso de la puntuación -puntos (Ppunto), comas (P-coma), mayúscula inicial de oración (P-may) y signos complejos (Psignos compl)- y la ausencia de encadenamientos (ME0). En este grupo se asocian fundamentalmente $(90 \%)$ alumnos de los séptimos grados de la Escuela Céntrica (EC7) y Hogar (EH7).

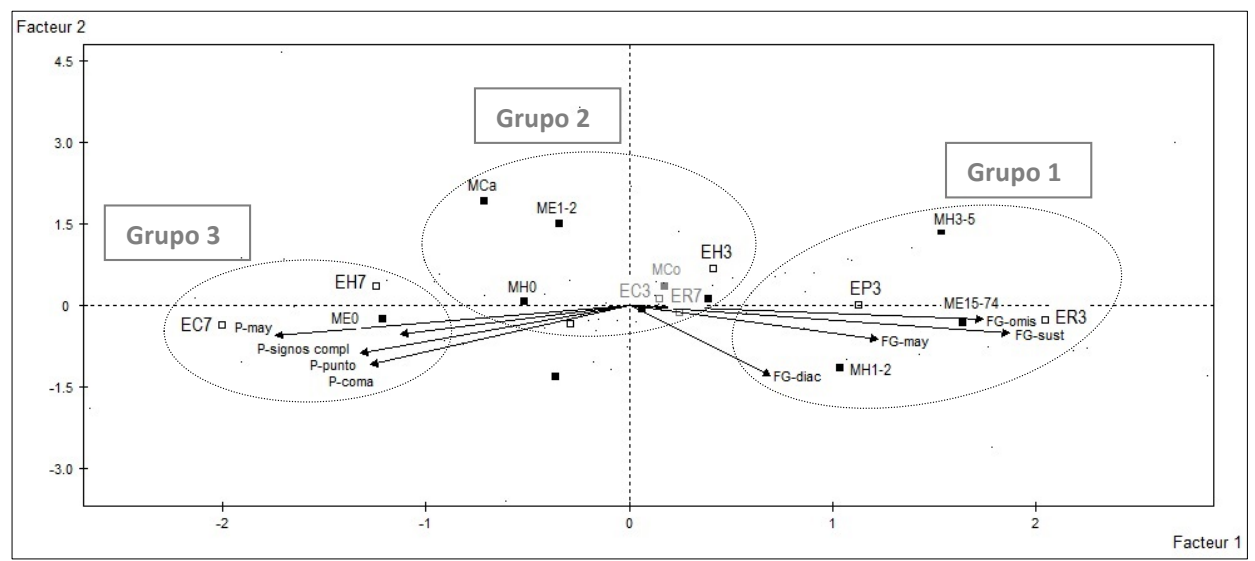

Figura 5. Nivel morfo-ortográfico. Primer plano factorial del Análisis de Correspondencias Múltiples: Proyección de las categorías y los grados escolares bien representados.

Nota: Los códigos utilizados para identificar las categorías fueron presentados en la Tabla 5 y los respectivos para los grados en la Tabla 1. 


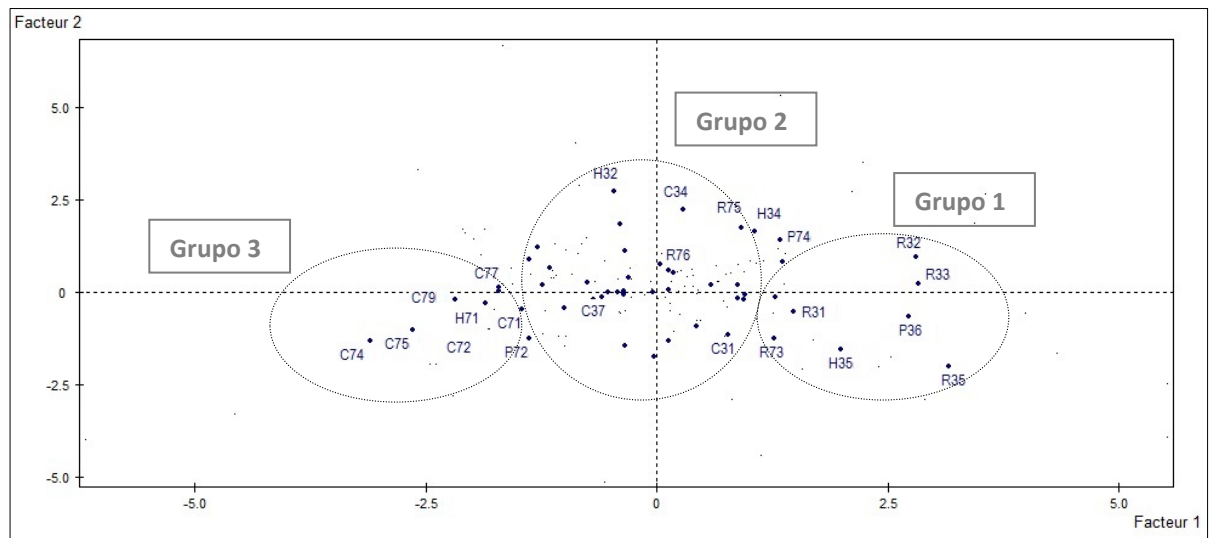

Figura 6. Nivel morfo-ortográfico. Primer plano factorial del Análisis de Correspondencias Múltiples: Proyección de los textos de los alumnos bien representados.

\section{Discusión}

Los tres grupos identificados a partir del análisis del nivel textual dieron cuenta de cierta progresión en el dominio de la estructura del texto narrativo escrito y que implica la explicitación de información, relacionada con aspectos evolutivo-educativos y también socioculturales. Así, alumnos de tercer grado de las dos escuelas de jornada simple con población de sectores socioeconómicos bajos y marginados se caracterizaron por textos con estilo descriptivo o que no llegaban a desarrollar inconclusos o que no constituían un relato (Grupo 1). Alumnos de tercer grado de la escuela rural con apoyo escolar incorporaron algunos componentes característicos de la narrativa en un marco testimonial de la intimidad, construyendo estructuras textuales con impronta copulativa (Grupo 2). Y alumnos de séptimo grado y de ambos grados de la escuela de doble jornada con población de sectores socioeconómicos medios y altos construyeron relatos ficcionales con explicitación de gran cantidad de componentes de la estructura narrativa y secuencialidad temporo-causal, acercándose a las características de las narrativas prototípicas (Grupo 3). La progresión señalada difiere de lo encontrado por Christie y Derewianka (2008), aunque las características de los textos de los grupos 1 y 2 presentan coincidencias con lo encontrado por Salvador Mata (1999). Esto podría vincularse con las distintas poblaciones de estudio: mientras Christie y Derewianka (2008) analizaron textos identificados por los docentes como 'buenas' narrativas producidas por niños entre 6 y 12 años, Salvador Mata (1999) comparó los textos de alumnos con distinto desempeño escolar de tercer y cuarto grado.

En el nivel lexicogramatical identificamos cinco grupos que dieron cuenta de patrones lexicogramaticales que configuraron tres estilos lingǘsticos. Estos estilos difieren entre alumnos con distintas características socioculturales. En los textos de alumnos de sectores socioeconómicos periférico-marginados cobraron especial 
relevancia las formulaciones que contextualizaban las acciones (cómo, dónde, cuándo), definiendo las circunstancias particulares en que éstas se producían (Grupo 1). En los textos de alumnos de zonas rurales y de sectores socioeconómicos bajos y periféricos cobraron especial relevancia los grados y tipos de intervención de los roles participantes requeridos por los verbos (Grupos 2 y 3). En los textos del Grupo 2 los sucesivos hechos eran movilizados por parte de las entidades animadas que ejecutaban acciones en las que otros participantes animados e inanimados se veían afectados, en tanto que en el Grupo 3 los participantes decían, escuchaban y experimentaban, otorgando a la narrativa una impronta dialógica. Alumnos de sectores socioeconómicos medios y altos mostraron una impronta relacional entre los sucesos que construían las vicisitudes que daban origen a sus relatos, explicitando la articulación entre ellos (Grupos 4 y 5). En el caso de los alumnos del Grupo 4, los relatos avanzaron a través de la sucesión de acciones articuladas copulativamente, mientras que en el caso del Grupo 5 los alumnos jerarquizaron y expandieron la información por medio de variadas formas de subordinación y de opciones verbales, incorporando distintos planos temporales y aspectuales y otorgando a los relatos un alto grado de textura.

Los tres grupos identificados a partir del análisis morfo-ortográfico mostraron cierta progresión evolutivo-educativa y a través de los establecimientos escolares respecto del dominio de aspectos normativos de la escritura. Alumnos de tercer grado de las dos escuelas con población de sectores socioeconómicos bajos y marginados de jornada simple revelaron severas dificultades para poner en juego convenciones de producción básicas a nivel de la delimitación y codificación gráfica de la palabra (Grupo 1). Alumnos de tercer grado de sectores socioeconómicos medio-altos y de zonas rurales con apoyo escolar, y de séptimo grado de sectores socioeconómicos bajos mostraron manejar con mayor estabilidad la palabra como unidad de la lengua escrita (con errores puntuales en la delimitación gráfica y sin caracterizarse por errores ortográficos). Sus dificultades en el establecimiento de concordancias morfosintácticas indican intentos de integración de las palabras en estructuras mayores (Grupo 2). Y alumnos de séptimo grado de las escuelas con población de sectores socioeconómicos medios y altos y de zonas rurales con apoyo escolar mostraron dominio de la palabra como unidad formal y de aspectos de la composición textual, como la puntuación, dimensión que da cuenta de la delimitación de segmentos textuales (Grupo 3). La progresión señalada en este nivel se encuentra en coincidencia con estudios previos (Álvarez, 1996; Ferreiro \& Zucchermaglio, 1996; Sotomayor et al., 2013).

\section{CONCLUSIONES}

Estos resultados mostraron que los alumnos de las escuelas situadas en zonas periféricas y marginadas y que no contaban con doble escolaridad ni apoyo escolar, fueron los alumnos que dieron cuenta de un menor dominio del sistema de escritura respecto de los otros grados y escuelas, encontrándose con dificultades para la 
codificación de la escritura y para construir estructuras narrativas. En contraste, alumnos de las escuelas con doble jornada y apoyo escolar mostraron un mayor dominio del sistema de escritura en los dos momentos de la escolarización considerados. Entre ellos, los que asistían a tercer grado integraron algunos de los componentes del esquema narrativo, en tanto que aquellos que asistían a séptimo grado fueron quienes finalizaban el nivel primario con un mayor dominio de la escritura y produjeron textos cercanos al esquema narrativo prototípico. Esto último muestra la responsabilidad que cabe a las estrategias escolares, en especial en poblaciones escolares en las que la escritura tiene una menor pregnancia en las actividades cotidianas.

La tensión entre las prescripciones y las elecciones para la construcción textual se jugó diferencialmente en cada nivel lingüístico. En las progresiones evolutivoeducativas señaladas encontramos la orientación prescriptiva netamente en el nivel morfo-ortográfico y parcialmente en el nivel textual, a partir de producciones textuales que no se sitúan en el esquema narrativo. En cambio, la explotación de alternativas se juega en el nivel textual en las variaciones en los relatos producidos una vez que se alcanza a ingresar en las convenciones que regulan la estructura narrativa, y sin duda en el nivel lexicogramatical, donde la tensión entre la convencionalidad y la elección se pone especialmente en evidencia. Si bien el grado de dominio de las pautas prescriptivas circunscriben el arco de opciones lingüístico-discursivas posibles para desplegar plenamente las elecciones personales, los patrones lexicogramaticales encontrados configuraron estilos diferenciados basados en el uso de los recursos disponibles para estos niños.

En suma, los resultados nos permitieron captar diversos grados y formas de apropiación de la escritura narrativa, atendiendo a esta doble perspectiva de ajuste convencional y de exploración y explotación de horizontes de elección, a la vez que evidenciaron la complejidad representacional de la narrativa escrita individual (Nelson, 2014), sin un interlocutor que ayude a sostener y avanzar en la construcción textual (Rosemberg et al., 2011). Así, en tanto la narrativa escrita constituye una instancia de elaboración y representación lingüístico-discursiva de la experiencia, a pesar de que las convenciones restringen el arco de variaciones socioculturales, tal variación también se manifiesta en las elecciones que hacen los alumnos en el marco de tales convenciones.

Para finalizar, consideramos que el triple enfoque adoptado nos permitió conocer distintas resoluciones posibles a la tarea, contribuyendo a profundizar la noción de la escritura como práctica sociocultural atravesada por múltiples dimensiones lingüísticodiscursivas. Por un lado, permitió vislumbrar aquellos aspectos que presentan dificultades persistentes especialmente para los alumnos de sectores socioeconómicos bajos y marginados, incluso en su tercer año de educación primaria obligatoria. Por otro, mostró aquellos recursos que se vinculan con la identidad de los alumnos y que 
resultan potentes como base para amplificar las posibilidades de expresión escrita de los alumnos.

Creemos que estos resultados aportan información acerca de los recursos desplegados y las dificultades a las que se enfrentan alumnos de primaria de un amplio arco sociocultural en la producción narrativa escrita. La integración deliberada de estos recursos junto a la atención a estas dificultades podría favorecer la expresión personal en el marco de las propias pautas culturales, así como la apropiación de las reglas básicas de la escritura y las pautas que regulan el género, imprescindibles para el reconocimiento de y el diálogo entre las pautas y estilos personales y familiares (o comunitarios), y aquellas vinculadas a la cultura letrada (y, por extensión, escolar), así como el fortalecimiento de los niños como escritores autorizados.

Cabe mencionar algunas limitaciones que podrían abordarse en futuras investigaciones.

A fin de establecer el grado de robustez de los resultados conforme la variable socioeducativa, sería recomendable ampliar el estudio incorporando otros establecimientos escolares con similares características a los cuatro seleccionados.

Dado que la narrativa constituye un género utilizado en gran variedad de situaciones y con diversos fines, sería de interés explorar cómo resuelven los alumnos la tensión entre ambas orientaciones al producir otros tipos de narrativas escritas, y, en particular, conocer en qué medida los estilos identificados se mantienen o se identifican otros.

Por último, en este estudio no hemos ahondado en los contextos habituales de producción escrita de los alumnos, sea formales o informales. Sin duda, un mayor conocimiento de las prácticas de escritura de los niños dentro y fuera de las escuelas contribuiría a una mejor comprensión del sentido y la configuración de los patrones y estilos lingüístico-discursivos de los que se dio cuenta en este trabajo. 


\section{REFERENCIAS BIBLIOGRÁFICAS}

Adam, J. (1992). Les textes. Types et prototypes. París: Nathan.

Adam, J. (1999). Linguistique textuelle. Des genres de dicours aux textes. París: Nathan.

Álvarez, G. (1996). Conexión textual y escritura en narraciones escolares. Onomazein, 1, 11-29.

Bajtin, M. (1985). Estética de la creación verbal. México: Siglo XXI.

Bécue-Bertaut, M. \& Pagès, J. (2000). Analyse Factorielle Múltiple intra-tableux. Application à l' analyse simultanée de plusieurs questions ouvertes. En M. Rajman \& J. C. Chappelier (Eds.), Sème Journées Internationales d'Analyse statistique de Donnée Textuelles (pp. 425-432). Lausanne: EPFL.

Beers, S. \& Nagy, W. (2011). Writing development in four genres from grades three to seven: Syntactic complexity and genre differentiation. Reading and Writing, 24, 183-202.

Benzécri, J. (1976). L'Analyse des Données. París: Dunod.

Berman, R. \& Slobin, D. (1994). Relating events in narrative: A Crosslinguistic developmental study, Volume 1. Nueva Jersey: Lawrence Erlbaum Associates.

Biber, D. (1988). Variation across speech and writing. Cambridge: Cambridge University Press.

Bosque, I. \& Demonte, V. (Eds.) (1999). Gramática descriptiva de la lengua española. Madrid: R.A.E.-Espasa Calpe.

Bruner, J. S. (1991). The narrative construction of reality. Critical Inquiry, 18(1), 1-21.

Bruner, J. S. (2002). Making stories: Law, literature, life. Nueva York: Farrar, Strauss and Giroux.

Christie, F. \& Derewianka, B. (2008). School discourse. Learning to write across the years of schooling. Londres/Nueva York: Continuum.

Crivisqui, E. (1993). Análisis factorial de correspondencias: Un instrumento de investigación en ciencias sociales. Asunción: Editorial de la Universidad Católica de Asunción.

Donald, M. (1993). Précis of Origins of the modern mind: Three stages in the evolution of culture and cognition. Behavioural and Brain Sciences, 15, 737-791.

Dowty, D. (1991). Thematic proto-roles and argument selection. Language, 67(3), 547619. 
Ferreiro, E. \& Teberosky, A. (1979). Los sistemas de escritura en el desarrollo del niño. Buenos Aires: Siglo XXI.

Ferreiro, E. \& Zucchermaglio, C. (1996). Children's use of puctuation marks: The case of quoted speech. En C. Pontecorvo, M. Orsolini, B. Burge \& L. Resnick (Eds.), Children's Early Text Construction. Moahwah (pp. 177-205). NJ: Lawrence Erlbaum Associates.

Fitzgerald, J. \& Shanahan, T. (2000). Reading and writing relations and their development. Educational Psychologist, 35(1), 39-50.

García Guzmán, A. \& Salvador Mata, F. (2012). La competencia narrativa de alumnos de etnia gitana, escolarizados en Educación Primaria. Un estudio de caso. Revista de Investigación en Educación, 10(1), 144-157.

García Negroni, M. (2010). Escribir en español: Claves para una corrección de estilo. Buenos Aires: Santiago Arcos.

Givón, T. (2001). Syntax: An introduction (Vol. 1). Ámsterdam/Filadelfia: John Benjamins.

González Calvo, J. (1999). Sobre la palabra y las clases de palabras. Revista Española de Lingüistica, 30(2), 309-329.

Halliday, M.A.K. (1994). An introduction to Functional Grammar. Nueva York: St Martin's Press.

Iparraguirre, M. S. (2013). Escritura, variación lingüistica y escolarización. Concepciones de docentes y producciones de alumnos en la zona andina rionegrina. Tesis doctoral, Universidad de Buenos Aires, Facultad de Filosofía y Letras, Buenos Aires, Argentina.

Junco, M. (1999). La evaluación del discurso narrativo escrito en el nivel elemental de enseñanza. Milenio, 3, 213-244.

Kamberelis, G. (1999). Genre development and learning: Children writing Stories, Science Reports, and Poems. Research in the Teaching of English, 33, 403-460.

Klein, I. (2007). La narración. Buenos Aires: Eudeba.

Knudson, R. E. (1995). Writing experiences, attitudes, and achievement of first to sixth graders. The Journal of Educational Research, 89(2), 90-97.

Labov, W. (1972). Language in the inner city. Philadelphia: University of Pennsylvania Press. 
Lane, S. \& Lewandowski, L. (1994). Oral and written compositions of students with and without learning disabilities. Journal of Psychoeducational Assessment, 12(2), 142-153.

Lebart, L., Morineau, A. \& Piron, M. (1995). Statistique exploratoire multidimensionnelle. Dunod: París.

Miller, P. J., Koven, M. \& Lin, S. (2011). Language socialization and narrative. En A. Duranti, E. Ochs \& B.B. Schieffelin (Eds.), The Handbook of Language Socialization (pp. 190-208). Malden: Wiley-Blackwell.

Maimone, M. C. \& Edelstein, P. (2004). Acerca de la dignidad en el proceso educativo. Buenos Aires: Stella - La Crujía.

Ministerio de Cultura y Educación de la Nación (2010). Operativo Nacional de Evaluación 2010. $3^{\circ}$ y $6^{\circ}$ año de la Educación Primaria. Informe de Resultados [en línea]. Disponible en: http://portales.educacion.gov.ar

Montague, M., Maddux, C. \& Dereshiwsky, M. (1990). Story grammar and comprehension and production of narrative prose by students with learning disabilities. Journal of Learning Disabilities, 23(3), 190-197.

Nelson, K. (1996). Language in cognitive development. The emergence of the mediated mind. Cambridge: Cambridge University Press.

Nelson, K. (2014). Pathways from infancy to the community of shared minds / El camino desde la primera infancia a la comunidad de mentes compartidas. Infancia y Aprendizaje: Journal for the Study of Education and Development, 37(1), 124.

Ochs, E. \& Capps, L. (1996). Narrating the self. Annual Review of Anthropology, 25, 1943.

Peterson, Sh. (2001). Gender identities and self-expression in classroom narrative writing. Language Arts, 8(5), 451-457.

R.A.E. (2011). Ortografía de la lengua española. Buenos Aires: Espasa.

Rosemberg, C., Silva, M. \& Stein, A. (2011). Narrativas infantiles en contexto: Un estudio en hogares de barrios urbano marginados de Buenos Aires. Revista del Instituto de Investigaciones en Ciencias de la Educación, 28, 135-154.

Salvador Mata, F. (1999). Habilidades narrativas de alumnos de educación primaria en la producción de textos escritos. Enseñanza, 17-18, 145-163. 
Simon, H. A. \& Hayes, J. R. (1979). Understanding written problem instructions. En H. A. Simon (Ed.), Models of thought (pp. 127-156). New Haven: Yale University Press.

Snow, C. (2006). What counts as Literacy in early childhood? En K. McCartney \& D. Phillips (Eds.), Handbook of Early Child Development (pp. 274-294). Oxford: Blackwell.

Sotomayor, C., Molina, D., Bedwell, P. \& Hernández, C. (2013). Caracterización de problemas ortográficos recurrentes en alumnos de escuelas municipales chilenas de $3^{\circ}, 5^{\circ}$ y $7^{\circ}$ básico. Revista Signos. Estudios de Lingüistica, 46(81), 105131.

Stein, N. L. \& Glenn, C. G. (1979). An analysis of story comprehension in elementary school children. En R. O. Freedle (Ed.), New directions in discourse processing, Volume 2 (pp. 53-120). Norwood: Ablex.

Peterson, C. (1994). Narrative skills and social class. Canadian Journal of Education, 19(3), 251-269.

Tolchinsky, L. (2006). The emergence of writing. En Ch. MacArthur, S. Graham \& J. Fitzgerald (Eds.), Handbook of Writing Research (pp. 83-95). Nueva York: The Guilford Press.

van Dijk, T. A. \& Kintsch, W. (1983). Strategies of discourse comprehension. Nueva York: Academic Press.

\section{NOTA}

${ }^{1}$ Agradecemos a las escuelas participantes por abrirnos sus puertas para realizar este estudio. Este trabajo contó con el apoyo de la Universidad Nacional del Comahue (Argentina) a través del Proyecto C-107, del Consejo Nacional de Investigaciones Científicas y Técnicas (CONICET, Argentina) a través del proyecto PIP 112-201301-00142 y de la Agencia Nacional de Promoción de la Ciencia y la Técnica (ANPCYT, Argentina) a través del proyecto PICT 2014-1016. 